\title{
Avaliação do perfil laboratorial de idosos com fratura de fêmur proximal por mecanismo de baixa energia*
}

\section{Evaluation of the Laboratorial Profile of Elderlies with Low-Energy Proximal Femur Fracture}

\author{
Marcelo Baggio $^{1}$ Daniel Teixeira de Oliveira ${ }^{2}$ Renato Locks ${ }^{1}$
}

${ }^{1}$ Hospital Regional de São José Dr. Homero de Miranda Gomes, São José, SC, Brasil

2 Cirurgia do Joelho, Escola Paulista de Medicina, Universidade Federal

Endereço para correspondência Marcelo Baggio, Rua Adolfo Donato da Silva, Hospital Regional de São José Dr. Homero de Miranda Gomes, s/n. de São Paulo, São Paulo, SP, Brasil

Rev Bras Ortop 2019;54:382-386.

\section{Resumo \\ Palavras-chave \\ - mortalidade \\ - fraturas do quadril \\ - vitamina D \\ - albuminas \\ - cálcio}

\section{Abstract}

Objetivo Avaliar o perfil laboratorial de idosos com fratura de fêmur proximal e verificar a relação dos dados com o desfecho da própria fratura e com o desfecho óbito. Métodos Estudo transversal de pacientes admitidos na emergência ortopédica de um hospital referência, entre os meses de fevereiro e abril de 2017, com fratura de fêmur proximal, por mecanismo de baixa energia, sendo coletados exames laboratoriais e de imagem. Foram excluídos do estudo pacientes com suspeita ou confirmação de fratura patológica.

Resultados Foram avaliados 66 indivíduos, sendo 44 mulheres, todos com idade superior a 60 anos. A fratura transtrocantérica apresentou maior incidência no estudo (36). Alterações do hormônio da paratireoide (PTH) e da albumina foram significativos para óbito $(p \leq 0,05)$. O tempo de internação não foi fator significativo para óbito. Conclusões Alterações laboratoriais não estavam relacionadas ao desfecho de óbito. A albumina pode estar relacionada ao risco de óbito. Nenhum resultado laboratorial foi apontado como facilitador na geração de fraturas de fêmur proximal. Mais estudos são necessários para poder entender melhor a influência do quadro laboratorial do paciente na ocorrência de fraturas e suas consequências.

Objective To evaluate the laboratory profile of elderly patients with proximal femoral fractures and to verify the relation between the data and the outcome to the fractures themselves and the occurrence of death.

Methods Cross-sectional study of patients admitted to the orthopedic emergency of a reference hospital between February and April 2017, with proximal femoral fracture, by low energy mechanism, when laboratory and imaging exams were collected. Excluded from the study were patients with suspected or confirmed pathological fracture.

\footnotetext{
* Trabalho feito no Hospital Regional de São José Dr. Homero de Miranda Gomes, São José, SC, Brasil.

(D) Marcelo Baggio's ORCID is https://orcid.org/0000-0003-4070-7919.
}

recebido

21 de Março de 2018 aceito 17 de Julho de 2018
DOI https://doi.org/

$10.1055 / \mathrm{s}-0039-1693667$. ISSN 0102-3616.
Copyright $\odot 2019$ by Sociedade Brasileira License terms de Ortopedia e Traumatologia. Published by Thieme Revnter Publicações Ltda, Rio de Janeiro, Brazil 
Keywords

- mortality

- hip fractures

- vitamin D

- albumins

- calcium
Results Sixty-six individuals were evaluated, 44 of whom were women, all 60 years old. The transtrochanteric fracture had a higher incidence in the study (36). Alterations in the levels of parathyroid hormone (PTH) and albumin were significant for death $(p \leq 0.05)$. The length of hospital stay was not a significant factor for death.

Conclusions Laboratory abnormalities were not related to the outcome of death. Albumin may be related to the risk of death. No laboratory result was pointed out as a facilitator in the generation of proximal femoral fractures. More studies are necessary to better understand the laboratory influence on fractures and their consequences.

\section{Introdução}

O envelhecimento da população é uma realidade que vem sendo observada recentemente. Ao avaliar os últimos 20 anos, observou-se um aumento de $5 \%$ em pessoas com idade $\geq 60$ anos, chegando a aproximadamente $12 \%$ do total de indivíduos vivos. Essa estatística leva a maior atenção que é necessária com essa faixa etária, entre elas a fratura do terço proximal do fêmur, ditas fraturas do quadril. ${ }^{1-3}$

As fraturas do terço proximal do fêmur em pacientes idosos são causas de elevada morbimortalidade. Em um estudo observacional realizado em 2015 por Daniachi et $\mathrm{al}^{4}{ }^{4}$ observou-se que dos 113 pacientes, 92,9\% sofreram fraturas associadas a traumas de baixa energia, sendo que, em sua maioria, elas ocorriam dentro da própria casa por queda da própria altura. Dentro dos principais fatores de risco associados, foi observado em uma revisão de literatura, que as variáveis que mais estavam associadas à fratura e/ou ao óbito após a fratura eram idade avançada e alterações do metabolismo do cálcio, albumina e vitamina D. ${ }^{3-7}$

Um estudo chinês realizado por $\mathrm{Li}$ et al mostrou que pacientes com fratura de fêmur proximal apresentavam níveis de cálcio sérico em valores abaixo da referência. ${ }^{8}$ Na mesma linha, Ramason et $\mathrm{al}^{9}$ reportaram que estes pacientes apresentavam deficiência ou insuficiência de vitamina D, culminando em óbito no caso de alguns fraturados. Outro estudo, apontou que a alteração de cálcio e vitamina $\mathrm{D}$ podem influenciar nos resultados de hormônio da paratireoide (PTH), o qual também foi relacionado ao óbito. ${ }^{10}$ Koval et a ${ }^{11}$ acreditam que o estado nutricional do paciente pode ter influência na mortalidade, e a hipoalbuminemia pode ser um marcador.

A relação de parâmetros laboratoriais e radiológicos de pacientes com fratura do terço proximal do fêmur tem sido investigada; no entanto, poucas pesquisas têm abordado essa temática. O objetivo deste estudo é traçar o perfil osteometabólico de pacientes idosos com fratura do quadril e avaliar possíveis causas que podem estar facilitando o desfecho da fratura.

\section{Material e Métodos}

Foi realizado um estudo transversal com 66 pacientes. Os indivíduos que compuseram a amostra preenchiam os seguintes critérios de inclusão: ter sofrido fratura do terço proximal do fêmur, idade superior a 60 anos e procura pelo serviço oferecido em um hospital referência em ortopedia e traumatologia, no período compreendido entre os dias 02 de fevereiro de 2017 até o dia 02 de abril de 2017.

O trabalho foi aprovado pelo comitê de ética da instituição envolvida.

Foram excluídos os pacientes cujos exames laboratoriais não foram realizados ou registrados no sistema do hospital e pacientes com histórico, suspeita ou confirmação de fratura patológica causadas por tumores.

As informações desse grupo de indivíduos foram coletadas a partir do prontuário eletrônico do referido hospital conforme autorização do guardião de prontuários da instituição. As variáveis analisadas compreenderam: sexo, idade, tipo de fratura, cálcio sérico, PTH sérico, 25-hidroxi-vitamina D sérica e albumina sérica.

Os exames laboratoriais foram coletados na chegada do paciente ao atendimento hospitalar e foram encaminhados para um único laboratório, que fez a análise de todas as amostras desta pesquisa.

Os dados coletados foram digitados em planilhas do programa Excel (Microsoft Corp, Redmond, WA, EUA) e em seguida exportado para o programa SPSS (Statistical Package for the Social Sciencies) Versão 15.0 (SPSS Inc., Chicago, IL, EUA) para a análise. Os dados foram apresentados utilizando estatística descritiva, sendo que as variáveis qualitativas foram expressas por meio de números absolutos e frequências, e as variáveis quantitativas por meio de média e desvio padrão. As variáveis categóricas foram testadas pelo teste qui quadrado ou teste exato de Fisher, quando apropriado, e as variáveis numéricas foram testadas pelo teste $t$ de Student. 0 nível de significância estabelecido foi o valor $p \leq 0,05$.

\section{Resultados}

No presente estudo foram avaliados 66 indivíduos com idade 60 anos. Conforme demonstrado na - Tabela $\mathbf{1}$, $66,7 \%(n=44)$ eram dos pacientes eram do sexo feminino; a média de idade observada foi de 78,8 $(n=66)$ com desvio padrão (DP) de 9,2. Dos indivíduos estudados, $15,2 \%(n=10)$ foram a óbito durante o período de internação e os demais tiveram alta após o tratamento hospitalar da fratura.

Os exames complementares solicitados na admissão do paciente estão relacionados na $\mathbf{- T a b e l a ~} \mathbf{2}$, enquanto que sua associação com o desfecho óbito foi apresentada na - Tabela 3.

Quando comparadas as médias de exames laboratoriais com os desfechos óbito e não óbito, observou-se que os valores de PTH e albumina foram estatisticamente significativos 
Tabela 1 Características sociodemográficas e clínicas dos pacientes atendidos no período de estudo

\begin{tabular}{|l|l|l|l|}
\hline \multirow{3}{*}{ Sexo } & Feminino & N & $\%$ \\
\cline { 2 - 4 } & Masculino & 44 & 66,7 \\
\hline \multirow{4}{*}{ Idade } & Média & 22 & 33,3 \\
\cline { 2 - 4 } & DP & 78,89 \\
\cline { 2 - 4 } & Mínimo & 9,206 \\
\cline { 2 - 4 } & Máximo & 61 \\
\hline \multirow{4}{*}{ Tipo de fratura } & Colo de fêmur & 97 & \\
\cline { 2 - 4 } & Transtrocantérica & 36 & 36,4 \\
\cline { 2 - 4 } & Subtrocantérica & 6 & 9,1 \\
\hline \multirow{3}{*}{ Óbito } & Não & 56 & 84,8 \\
\cline { 2 - 4 } & Sim & 10 & 15,2 \\
\hline
\end{tabular}

Abbreviation: DP, desvio padrão.

Tabela 2 Características das amostras de exames complementares na admissão do paciente

\begin{tabular}{|c|c|c|c|c|}
\hline & $\mathrm{N}$ & $\begin{array}{l}\text { Mínimo- } \\
\text { Máximo }\end{array}$ & Média & DP \\
\hline Índice de Dorr & 50 & $0,44-1,20$ & 0,77 & 0,13 \\
\hline Cálcio & 65 & $4,7-10,3$ & 8,61 & 1,02 \\
\hline PTH & 64 & $11,9-311,0$ & 63,50 & 52,35 \\
\hline $\begin{array}{l}\text { 25-hidroxi- } \\
\text { vitamina D }\end{array}$ & 65 & $6,3-72,4$ & 20,57 & 11,53 \\
\hline Albumina & 55 & $1,7-4,5$ & 3,25 & 0,64 \\
\hline
\end{tabular}

Abbreviations: DP, desvio padrão; PTH, hormônio da paratireoide.
( $p \leq 0,05)$, apresentando o PTH uma diferença de média de $58,0 \mathrm{pg} / \mathrm{mL}$ entre os que não foram a óbito para 93,1 pg/mL entre os que foram; quanto à albumina, os que foram a óbito apresentaram média de $2,7 \mathrm{pg} / \mathrm{mL}$ e os que não foram, $3,3 \mathrm{pg} / \mathrm{mL}$.

O tempo de internação não foi causa determinante para o óbito na amostra analisada.

Os valores laboratoriais de vitamina D não apresentaram variações quando comparados entre os sexos.

Quando comparadas as médias dos exames laboratoriais com os tipos de fratura, observa-se que não houve diferença estatisticamente significativa $(p>0,05)$ entre elas, conforme demonstrado na - Tabela 4.

Quando comparadas as variáveis de classificação do índice de Dorr e tipo de fratura, observa-se que não houve associação ( $p=0,828)$. Da mesma forma, o tipo de fratura não mostrou associação com o óbito $(p=0,555)$ e com o sexo $(p=0,191)$.

\section{Discussão}

Pesquisas avaliando a associação entre fraturas de quadril e resultados de exames laboratoriais são um tema que vem sendo estudado a fim de tentar estimar possíveis causas e assim evitar as consequências desta associação. ${ }^{9,10,12}$ Nos 66 indivíduos avaliados, em sua maioria do sexo feminino, esse trabalho apontou que a fratura transtrocantérica foi a mais frequente na faixa etária estudada (indivíduos 60 anos de idade). Não houve relação entre o tipo de fratura e desfecho óbito. Em relação aos exames laboratoriais, foi observado que os pacientes que foram a óbito apresentavam níveis elevados de PTH e redução dos níveis de albumina quando comparados aos pacientes que não foram a óbito. 0 tempo de internação não apresentou relação com a mortalidade durante a internação.

Tabela 3 Comparação das médias dos exames laboratoriais na amostra quando comparados à variável de desfecho (óbito e não óbito)

\begin{tabular}{|c|c|c|c|c|c|c|c|c|c|}
\hline & & Cálcio & $p$ & PTH & $p$ & 25-hidroxi-vitamina D & $p$ & Albumina & $p$ \\
\hline \multirow[t]{2}{*}{ Não óbito } & Média & 8,689 & \multirow[t]{4}{*}{0,17} & 58,015 & \multirow[t]{4}{*}{0,05} & 21,184 & \multirow[t]{4}{*}{0,32} & 3,324 & \multirow[t]{4}{*}{0,01} \\
\hline & $\mathrm{DP}$ & 0,850 & & 43,970 & & 12,134 & & 0,601 & \\
\hline \multirow[t]{2}{*}{ Óbito } & Média & 8,210 & & 93,140 & & 17,22 & & 2,683 & \\
\hline & $\mathrm{DP}$ & 1,716 & & 81,516 & & 6,88 & & 0,722 & \\
\hline
\end{tabular}

Abbreviations: DP, desvio padrão; PTH, hormônio da paratireoide.

Tabela 4 Comparação das médias dos exames laboratoriais na amostra quando comparados à classificação da fratura

\begin{tabular}{|c|c|c|c|c|c|c|c|c|c|}
\hline Topografia da fratura & & Cálcio & $p$ & PTH & $p$ & 25-hidroxi-vitamina D & $p$ & Albumina & $p$ \\
\hline \multirow[t]{2}{*}{ Colo de femur } & Média & 8,742 & \multirow[t]{6}{*}{0,43} & 62,313 & \multirow[t]{6}{*}{0,91} & 23,829 & \multirow[t]{6}{*}{0,12} & 3,415 & \multirow[t]{6}{*}{0,36} \\
\hline & DP & 1,0176 & & 41,018 & & 14,2542 & & 0,6961 & \\
\hline \multirow[t]{2}{*}{ Transtrocantérica } & Média & 8,471 & & 65,644 & & 17,852 & & 3,176 & \\
\hline & $\mathrm{DP}$ & 1,0701 & & 62,3672 & & 9,3766 & & 0,6092 & \\
\hline \multirow[t]{2}{*}{ Subtrocantérica } & Média & 8,95 & & 56,133 & & 23,433 & & 3,100 & \\
\hline & $\mathrm{DP}$ & 0,7765 & & 31,9787 & & 7,5617 & & 0,5865 & \\
\hline
\end{tabular}

Abbreviations: DP, desvio padrão; PTH, hormônio da paratireoide. 
Em um estudo realizado em Guangzhou, China, Li et $\mathrm{al}^{8}$ observaram que dos pacientes internados com fratura de fêmur proximal (196), as fraturas intertrocantérica apresentaram valores de cálcio sérico abaixo do padrão de referência. Larrosa et al, ${ }^{13}$ em sua amostra com 128 pacientes com fratura de colo de fêmur e 196 com fratura intertrocantérica, não observou alteração laboratorial nos valores de cálcio no momento da admissão hospitalar. Nos estudos citados, o desfecho mortalidade não foi citado. Em nosso estudo, foi percebido que o perfil laboratorial de cálcio não teve significância estatística nas fraturas de fêmur proximal, e o desfecho para óbito também não se apresentou relevante.

No presente estudo, não foi observada relação significativa entre os desfechos óbito e não óbito, assim como entre os tipos variados de fraturas e o sexo do paciente, e o nível de vitamina D. Este perfil laboratorial vem sendo estudado e atribuído por alteração em pacientes com fratura de quadril. Ramason et $\mathrm{al}^{9}{ }^{9}$ em uma amostra de 412 pacientes fraturados, perceberam que 57,5\% dos pacientes hospitalizados apresentaram deficiência de Vitamina D; 34,5\% mostraram insuficiência do laboratório analisado e $8 \%$ evoluíram para óbito. Outra evidência desse estudo foi que os pacientes que viviam em casas de repouso apresentaram níveis menores de Vitamina D em sua análise admissional. Em outra análise com grupo e controle, Guerra et $\mathrm{al}^{14}$ avaliaram 110 pacientes com fratura de quadril, dentre os quais $54,5 \%$ apresentaram deficiência de vitamina D e $18,2 \%$ apresentaram níveis insuficientes. $O$ autor mostra que houve diferença estatisticamente significativa entre o grupo fratura e o grupo controle, para os pacientes com idade superior a 70 anos, o que não ocorreu em pacientes com idade entre 60 e 65 anos. Gumieiro et al, ${ }^{15}$ em uma análise com 88 pacientes com fratura de quadril, não observaram diferença entre os valores de vitamina $\mathrm{D}$ quando comparando os tipos de fraturas e capacidade de retornar a deambular.

Avaliando os níveis sérico de PTH, Madsen et al, ${ }^{10}$ em uma amostra com 562 fraturas de quadril, acreditam que o óbito esteja relacionado à soma das alterações laboratoriais nos níveis de vitamina D e cálcio. $O$ autor não afirma que a alteração única de PTH indica risco de óbito, mas que isso pode ser um indicador de saúde precária, gerando um fator de instabilidade que pode levar ao óbito. Di Monaco et $\mathrm{al}^{16}$ e Fischer et al ${ }^{17}$ corroboram a análise de que a alteração do PTH pode ser fator de risco para o óbito, mas devido a amostra pequena, torna-se um fator limitante. Na amostra deste estudo, observou-se que a alteração do PTH apresenta valores significativos para o óbito, entretanto, assim como Monaco et $\mathrm{al}^{16}$ e Fischer et $\mathrm{al}^{17}$ o tamanho da amostra pode ser limitador.

Koval et $\mathrm{al}^{11}$ correlacionaram a hipoalbuminemia com a mortalidade tardia e entendem que a queda laboratorial tem relação direta devido ao estado nutricional inadequado. Grimes et $\mathrm{al}^{18}$ não relacionaram o tempo de internação com o óbito em sua análise de 8.383 pacientes. Parker et al, ${ }^{19}$ em uma metanálise com 428 pacientes, demonstraram que o tratamento cirúrgico não diminui o risco de morte quando comparado ao tratamento conservador. Sakaki et al, ${ }^{1}$ em uma revisão da literatura, concluíram que existem quatro grandes fatores influentes na mortalidade, sendo eles idade avançada, associação de comorbidades diversas, sexo masculino e deficiências cognitivas. Em nossa análise, foi observado que alterações laboratoriais de albumina e PTH têm relação significativa com o óbito, enquanto o tempo de internação não apresentou significância.

É importante salientar que sem a vitamina D, apenas 10 a $15 \%$ do cálcio dietético será absorvido a nível intestinal, o que levará a uma elevação forçada do PTH e um efeito rebote a nível tubular renal, gerando a reabsorção de cálcio. Com o aumento do PTH, ocorre em associação a reabsorção óssea, através da estimulação forçada dos osteoclastos, levando ao quadro de osteopenia e osteoporose, aumentando o risco de fraturas. ${ }^{20}$ Um estudo com 3.270 idosos que receberam reposição de 800 UI de vitamina D e $1.200 \mathrm{mg}$ de cálcio em dose diária por 3 anos apresentou redução da incidência de fraturas de quadril em $43 \%{ }^{21}$ Em uma metanálise que comparou a reposição diária entre 400 UI e 700 a 800 UI de vitamina $\mathrm{D}$, foi observado que doses baixas não apresentam alteração no desfecho fratura, mas que doses elevadas (700 a 800 UI) reduziram em $26 \%$ o risco relativo de fratura do quadril. $^{22} \mathrm{O}$ exposto mostra que há íntima relação osteometabólica, onde analisar de forma isolada marcadores de PTH, vitamina $\mathrm{D}$ e cálcio pode gerar um resultado falso negativo e uma interpretação errônea sobre o laboratório. A relação dietética com a hipoalbuminemia está ligada às alterações já expostas e corrobora para o quadro de desnutrição e em complementação a queda de cálcio ingerido.

O perfil do paciente e a análise foram realizados após observar na literatura que essas eram as variáveis que obtiveram maiores estudos e complicações associadas ao óbito. A associação do tema com doenças cognitivas e múltiplas comorbidades clínica também foi citada na literatura, mas devido à dificuldade de realizar um seguimento multidisciplinar em nosso serviço, por questões científicas, foi optado por não realizar essa correlação. 0 presente estudo pode estar confinado a resultados limitados devido ao pequeno número de pacientes estudados e a falta de seguimento a longo prazo. A coleta de sangue para análise laboratorial foi realizada de forma semelhante para todos os pacientes, não sendo, portanto, uma fonte limitadora em seus resultados. A análise dos dados foi realizada por um único profissional treinado, não apresentando, também, desvio em sua análise. 0 tempo médio de internação pode apresentar resultados divergentes da literatura, sendo que, nesse serviço, a reserva de vaga de UTI, quando necessário, apresenta dificuldade devido ao grande número de pacientes politraumatizados admitidos.

\section{Conclusões}

O desfecho para óbito aponta a possibilidade de alterações no nível de albumina aumentarem o risco, possivelmente relacionado à desnutrição. O aumento do PTH, neste estudo, apresentou tendência ao óbito, mas, devido ao baixo número de pacientes estudados, esse resultado pode apresentar-se limitado. Análises osteometabólicas relacionados à fratura de quadril carecem de estudos mais aprofundados para complementar os resultados já descritos na literatura e poder entender um pouco melhor a relação dos resultados laboratoriais com as fraturas e suas consequências. 
Conflitos de Interesse

Os autores declaram inexistência de conflito de interesses na realização deste trabalho.

\section{Referências}

1 Sakaki MH, Oliveira AR, Coelho FF, Leme LEG, Suzuki I, Amatuzzi MM. Estudo da mortalidade na fratura do fêmur proximal em idosos. Acta Ortop Bras 2004;12(04):242-249

2 Mesquita GV, Lima M, Santos AM, Alves EL, Brito JNPO, Martins MC. Morbimortalidade em idosos por fratura proximal do fêmur. Texto Contexto Enferm 2009;18(01):67-73

3 Astur DC, Arliani GG, Balbachevsky D, Fernandes HJ, Reis FB. Fratura da extremidade proximal do fêmur tratadas no Hospital São Paulo/Unifesp: estudo epidemiológico. Rev Bras Med 2013;68 (04):11-15

4 Daniachi D, dos Santos Netto A, Ono NK, Guimarães RP, Polesello GC, Honda EK. Epidemiology of fractures of the proximal third of the femur in elderly patients. Rev Bras Ortop 2015;50(04):371-377

5 Rocha MA, Azer HW, Nascimento VDG. Evolução funcional nas fraturas da extremidade proximal do fêmur. Acta Ortop Bras 2009;17(01):17-21

6 Cauley JA, Lacroix AZ, Wu L, Horwitz M, Danielson ME, Bauer DC, et al. Serum 25-hydroxyvitamin D concentrations and risk for hip fractures. Ann Intern Med 2008;149(04):242-250

7 Uzoigwe CE, Venkatesan M, Smith R, Burnand HG, Young PS, Cheesman CL, et al. Serum lactate is a prognostic indicator in patients with hip fracture. Hip Int 2012;22(05):580-584

8 Li PF, Lin ZL, Pang ZH, Zeng YR. Does serum calcium relate to different types of hip fracture? A retrospective study. Chin J Traumatol 2016;19(05):275-277

9 Ramason R, Selvaganapathi N, Ismail NH, Wong WC, Rajamoney GN, Chong MS. Prevalence of vitamin d deficiency in patients with hip fracture seen in an orthogeriatric service in sunny singapore. Geriatr Orthop Surg Rehabil 2014;5(02):82-86

10 Madsen CM, Jørgensen HL, Lind B, Ogarrio HW, Riis T, Schwarz P, et al. Secondary hyperparathyroidism and mortality in hip fracture patients compared to a control group from general practice. Injury 2012;43(07):1052-1057
11 Koval KJ, Maurer SG, Su ET, Aharonoff GB, Zuckerman JD. The effects of nutritional status on outcome after hip fracture. J Orthop Trauma 1999;13(03):164-169

12 Roddam AW, Neale R, Appleby P, Allen NE, Tipper S, Key TJ. Association between plasma 25-hydroxyvitamin $\mathrm{D}$ levels and fracture risk: the EPIC-Oxford study. Am J Epidemiol 2007;166(11):1327-1336

13 Larrosa M, Gomez A, Casado E, Moreno M, Vázquez I, Orellana C, et al. Hypovitaminosis D as a risk factor of hip fracture severity. Osteoporos Int 2012;23(02):607-614

14 Guerra MT, Feron ET, Viana RD, Maboni J, Pastore SI, Castro CC. Elderly with proximal hip fracture present significantly lower levels of 25-hydroxyvitamin D. Rev Bras Ortop 2016;51(05):583-588

15 Gumieiro DN, Pereira GJ, Minicucci MF, Ricciardi CE, Damasceno ER, Funayama BS. Associations of vitamin D deficiency with postoperative gait and mortality among patients with fractures of the proximal femur. Rev Bras Ortop 2015;50(02):153-158

16 Di Monaco M, Vallero F, Di Monaco R, Tappero R, Cavanna A. 25hydroxyvitamin $\mathrm{D}$, parathyroid hormone, and functional recovery after hip fracture in elderly patients. J Bone Miner Metab 2006;24 (01):42-47

17 Fisher A, Goh S, Srikusalanukul W, Davis M. Elevated serum PTH is independently associated with poor outcomes in older patients with hip fracture and vitamin D inadequacy. Calcif Tissue Int 2009;85(04):301-309

18 Grimes JP, Gregory PM, Noveck H, Butler MS, Carson JL. The effects of time-to-surgery on mortality and morbidity in patients following hip fracture. Am J Med 2002;112(09):702-709

19 Parker MJ, Handoll HH, Bhargara A. Conservative versus operative treatment for hip fractures. Cochrane Database Syst Rev 2000; (04):CD000337

20 Holick MF. Vitamin D deficiency. N Engl J Med 2007;357(03): 266-281

21 Chapuy MC, Arlot ME, Duboeuf F, Brun J, Crouzet B, Arnaud S, et al. Vitamin D3 and calcium to prevent hip fractures in elderly women. N Engl J Med 1992;327(23):1637-1642

22 Bischoff-Ferrari HA, Giovannucci E, Willett WC, Dietrich T, Dawson-Hughes B. Estimation of optimal serum concentrations of 25hydroxyvitamin D for multiple health outcomes. Am J Clin Nutr 2006;84(01):18-28. [Erratum. Am J Clin Nutr 2006;84:1253 